\title{
A New Approach for Efficient Clustering Using CECM Algorithm in WSN
}

\author{
Gohil Sonal $^{1}$, Sumit Chaudhary ${ }^{2}$, S.B. Batra ${ }^{3}$ \\ Student, Computer engineering, IIST, Ahmedabad, India ${ }^{1}$ \\ HOD, Computer engineering, IIST, Ahmedabad, India ${ }^{2}$ \\ HOD, Electronics and Communication, IIST, Ahmedabad, India ${ }^{3}$
}

\begin{abstract}
In this paper the proposed work is to compare the properties of the nodes in the cluster on the basis of probability of their being retrieved by other cluster or network. All this nodes and clusters contains battery level, memory, reverse signal strength indication, multicast routing information cost , time to live new node and time to live cluster on the basis of expectation clustering and maximization clustering in wireless sensor networks.
\end{abstract}

Keywords: Expectation, maximization, cluster head, energy efficiency, k-means, data aggregation, WSN.

\section{INTRODUCTION}

A wireless sensor network consists spaciously distributed self-sufficient devices using sensors to monitor substantial or environmental conditions such as temperature, sound, vibration, pressure, motion or pollutants to collaboratively pass their data from the network to a destination or plunge where the data can be analysed and observed. A sensor node is a basic element in a sensor network, with on-board sensors, processor, memory, wireless modem and power supply. Sensor nodes contain reasonable battery power with few celestial spaces and processing power.

In a computer system, a cluster is a group of servers and other assets that act like a single system and enable high convenience and in some cases, load balancing and parallel processing. It is currently canvassing the Earth's magnetic environment and its interaction with the solar wind in three dimensions. Expectation and Maximization algorithm a habitual method to find maximum likelihood or maximum a posteriori (MAP) estimates of parameters in statistical models, where the model depends on unperceived latent variables.

$\mathrm{K}$-means algorithm aims to disseverance $\mathrm{n}$ observations into $\mathrm{k}$ clusters in which each observation belongs to the cluster with the nearest contemptible, serving as an archetype of the cluster. This results in a partitioning of the data space into glial cells. K-means clustering tends to find clusters of comparable ample extent, while the expectation-maximization mechanism allows clusters to have various shapes.

\section{LITERATURE REVIEW}

In data mining field nowadays there is a vast range of various fields like science research, statistical application, disaster area and War Zone. All this are related to mining of data, energy, memory, efficient techniques and data aggregation and data collection in WSN with calculation of cluster nodes, cluster head and other efficient techniques using data cube. A serviceable network is required to suffice the application quest. In addition, energy devastation of nodes is a great demur in order to maximize network endurance. Unlike other networks, it can be venturesome, very swank or even impossible to charge or replace kaput batteries due to the rancorous nature of encompassment.

The main focus of this gizmo is predominantly on duty cycling contrivance which represent the most reconcilable technique for energy saving and the data-driven passage that can be used to skyrocket the energy efficiency. We will make a review on some intercommunication protocols proposed for sensor networks. The work is how to poll the sensor nodes concretion to guarantee a full indemnity and to reduce energy devastation in a sensor network. During this paper there's a shot to allow a large analogize of the routing protocols in WSNs.

The Problem nascency does not require a prairie information about the number of clusters, and it gives a spontaneous way for superintendence missing values. We give a ceremonialistic statement of the clusteringaggregation quandary, we discuss related work, and we suggest a number of algorithms. For respective of the methods we provide unintelligible guarantees on the endowment of unravel.

\section{MECHANISM}

(1) Phase 1: calculation of efficient expectation clustering Step 1: cluster creation

Cluster-Creation ( ) 
\{

For (ss_id=0; ss_id $<\mathrm{N} ;$ ss_id++)

\{

If $(\mathrm{Bcon}>=\mathrm{Apr} \& \& \mathrm{Pm}>=\mathrm{Tm})$

$/ /$ Bcon = Battery consumption

// Apr =Present Cluster battery level

$/ / \mathrm{Pm}=$ Present memory

$/ / \mathrm{Tm}=$ Total cluster memory

\{

Node will not be Included in Cluster

\}

else if (TTLnew>TTLclst)

// TTLnew= Time To Live new node

// TTLclst=Time To Live cluster

\{

If ((MRICnew $>$ MRICclst) \&\& (RSSInew $<$ RSSIclst $) \& \&$

(Bandnew $>$ Bandclst))

// MRICnew= Multicast Routing Information Cost

//MRICclst=Multicast Routing Information Cost of cluster

//RSSInew=Reverse Signal Strength Indication of new

node

//RSSIclst =Reverse Signal Strength Indication of new

node

//Bandnew= Level Bandwidth new node

$/ /$ Bandclst= Level Bandwidth cluster

\{

No new node can join Cluster ( );

\}

else

\{

Join cluster;

\}

\}

\}

\}

step 2: cluster head selection on the basis of expectation clustering

for cluster 1 to cluster $\mathrm{k}$

\{

for node1 to node $\mathrm{n}$

\{

sump $=0$;

$1 \mathrm{lh}=0$;

for $(i=0 ; i<=n ; i++)$

\{

$$
\begin{gathered}
\delta_{i j}=\left(D_{i}-C_{j}\right)^{t} R^{-}\left(D_{i}-C_{j}\right) \\
P_{i}=\frac{W_{j}}{(2 \pi)^{p / 2}|R|} e^{\left[\frac{-1}{2} \delta_{i j}\right]}
\end{gathered}
$$

Sump $=\operatorname{sump}+P_{i} ;$

\}

$1 \mathrm{hh}=1 \mathrm{lh}+\ln (\mathrm{sump})$

$\mathrm{c}^{\prime}=\mathrm{c}^{\prime}+Y_{i} X_{i}$;

$\mathrm{w}^{\prime}=\mathrm{w}^{\prime}+\mathrm{B}^{\prime}$;

\}
\}

(2)phase 2: calculation of efficient maximization clustering

step 1: new node selection for maximization clustering

\section{NewlyArrivingNodeInWSN ( ) \\ \{ \\ Calculate the following factor}

Bcon[i], Apr[i], Pm[i], Bandnew[i], Bandclst[i], TTLnew[i],Tm[i], $\quad$ TTLclst[i], $\quad$ MRICnew[i], RSSInew[i],MRICclst[i], RSSIclst[i];

Calculate C (i1), C (i2), C (i3);

Calculate GCmin;

If (newly arrive node GCmin< cluster head GCmin)

Assign new node as cluster head;

\}

else

\{

Join cluster ( );

\}

Step 2: calculation of maximization clustering ClusterHeadAssignment ( )

\{

Total no of node $=$ n;

For (cluster1 to cluster $n$ )

\{

For $(\mathrm{i}=0 ; \mathrm{i}<\mathrm{n} ; \mathrm{i}++)$

\{

/*initialize parameter for cost evaluation*/

Bcon[i] $=\{\} ; / *$ Battery consumption at node $i^{*} /$

Apr[i] $=\{\} ; / *$ Present Cluster battery level at node $\mathrm{i} * /$

$\operatorname{Pm}[\mathrm{i}]=\{\} ; / *$ Present memory at node $\mathrm{i} * /$

$\operatorname{Tm}[\mathrm{i}]=\{\} ; / *$ Total cluster memory*/

TTLnew $[\mathrm{i}]=\{\} ; / *$ Time to Live new node $* /$

TTLclst $[\mathrm{i}]=\{\} ; / *$ Time to Live cluster*/

MRICnew $[\mathrm{i}]=\{\} ; / *$ Multicast Routing Information Cost at node $i * /$

MRICclst $[i]=\{\} ; / *$ Multicast Routing Information Cost of cluster*/

RSSInew $[\mathrm{i}]=\{\} ; / *$ Reverse Signal Strength Indication of new node*/

RSSIclst $[\mathrm{i}]=\{\} ; / *$ Reverse Signal Strength Indication of new node $* /$

Bandnew $[\mathrm{i}]=\{\} ; / *$ Level Bandwidth new node*/

Bandclst $[\mathrm{i}]=\{\} ; / *$ Level Bandwidth cluster*/

/* now calculate the cost for each node */

$\left.\mathrm{C}(\mathrm{i} 1)=\left(\operatorname{Apr}[\mathrm{i}]^{*} \mathrm{TTLnew}[\mathrm{i}] / \mathrm{Bcon}[\mathrm{i}]^{*} \mathrm{TTLclst}[\mathrm{i}]\right)\right)$

$\mathrm{C}(\mathrm{i} 2)=\left(\operatorname{Pm}[\mathrm{i}]^{*}\right.$ RSSInew $[\mathrm{i}] / \mathrm{Tm}[\mathrm{i}]^{*}$ RSSIclst $\left.[\mathrm{i}]\right)$

C

(MRICnew[i]*Bandnew[i]/MRICclst[i]*Bandclst[i] \}

Find out min. global cost for each node.

$\mathrm{GCmin}=\sum \mathrm{C}(\mathrm{i} 1)+\mathrm{C}(\mathrm{i} 2)+\mathrm{C}(\mathrm{i} 3)$ 
\}

$\min =0 ; \max =0$;

for $(i=1$ to $n)$

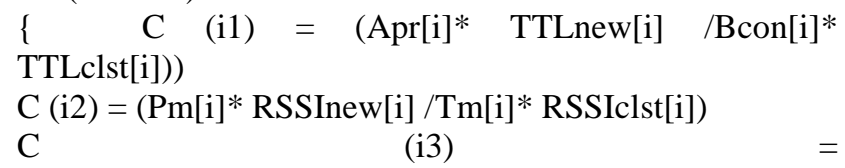

(MRICnew[i]*Bandnew[i]/MRICclst[i]*Bandclst[i] \}

$\mathrm{GCmin}=\mathrm{C}(\mathrm{i} 1)+\mathrm{C}(\mathrm{i} 2)+\mathrm{C}(\mathrm{i} 3)$;

If $(\max <\mathrm{GCmin})$

\{

$\max =\mathrm{GCmin} ;$

\}

else if (min $>$ GCmin)

\{

$\min =\mathrm{GCmin}$;

\}

\}

\}

\}

\section{CONCLUSION}

This paper applause for better and efficient techniques for cluster head selection and calculation of nodes of data cube on the basis of weight, memory and like that other things using clustering expectation and clustering maximization algorithm. It provides efficient techniques for energy saving in clustering with CECM algorithm in WSN with data cube aggregation.

\section{FUTURE SCOPE}

The proposed work provides efficient methods for energy in data aggregation but the field of data mining has various heterogeneous behavior in cluster node with ongoing advancement.

\section{REFERENCES}

[1] Chaudhary, Sumit, et al. "Energy Efficient Techniques for Data aggregation and collection in WSN." International Journal of Computer Science, Engineering and Applications 2.4 (2012): 37.

[2] Soua, Ridha, and Pascale Minet. "A survey on energy efficient techniques in wireless sensor networks." Wireless and Mobile Networking Conference (WMNC), 2011 4th Joint IFIP. IEEE, 2011..

[3] Rezaei, Zahra, and Shima Mobininejad. "Energy saving in wireless sensor networks." International Journal of Computer Science and Engineering Survey 3.1 (2012): 23 .

[4] Kaur, Baljinder, and Amandeep Kaur. "A survey of time synchronization protocols for wireless sensor networks." International Journal of Computer Science and Mobile Computing 2.9 (2013): 100-106..

[5] Gionis, Aristides, Heikki Mannila, and Panayiotis Tsaparas. "Clustering aggregation." ACM Transactions on Knowledge Discovery from Data (TKDD) 1.1 (2007): 4.

[6] Wang, Xi, Chunyu Yang, and Jie Zhou. "Clustering aggregation by probability accumulation." Pattern Recognition 42.5 (2009): 668-675.

[7] Hartigan, John A., and Manchek A. Wong. "Algorithm AS 136: A k-means clustering algorithm." Journal of the Royal Statistical Society. Series C (Applied Statistics) 28.1 (1979): 100-108.

[8] Kanungo, Tapas, et al. "An efficient k-means clustering algorithm: Analysis and implementation." IEEE transactions on pattern analysis and machine intelligence 24.7 (2002): 881-892.

[9] Celeux, Gilles, and Gérard Govaert. "A classification EM algorithm for clustering and two stochastic versions." Computational statistics \& Data analysis 14.3 (1992): 315-332..

[10] Hathaway, Richard J. "Another interpretation of the EM algorithm for mixture distributions." Statistics \& Probability Letters 4.2 (1986): 53-56..

[11] Ghahramani, Zoubin, and Geoffrey E. Hinton. The EM algorithm for mixtures of factor analyzers. Vol. 60. Technical Report CRGTR-96-1, University of Toronto, $1996 .$.

\section{BIOGRAPHIES}

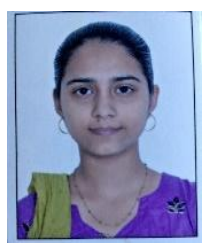

Gohil Sonal is pursuing BE in Computer Engineering from IIST, rajpur, kadi, Gujarat, India. She is currently doing her $8^{\text {th }}$ semester project in .Net language. Her interest of area is networking in WSN.

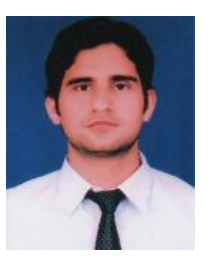

Sumit Chaudhary is working as Head of Department in CSE at Indrashil Institute of Science \& Technology, Cadila Group, Rajpur, Ahmedabad (Gujarat). $\mathrm{He}$ is pursuing Ph.D. from Uttaranchal University, Dehradun (Uttarakhand). He worked with various institutes like Uttaranchal Institute of Technology (UIT), Dehradun, Shri Ram Group of colleges, Muzaffarnagar (U.P.), IIMT Institute of Engineering \& Technology, Meerut (U.P.), INDIA including all that he has more than 7 year experience in teaching. He obtained his M-Tech (Computer Science \& Engineering) with Hons from Shobhit University and B-Tech (Computer Science \& Engineering) from SCRIET, Meerut (U.P.). During this short period of time, he has been supervised several dissertation of M.Tech students. He has been member of several academic and administrative bodies. During his teaching he has been coordinated many Technical fests and National Conferences at Institute and University Level. He has attended several seminars, workshops and conferences at various levels. His many papers are published in various national, international journals and conferences. His area of research includes Cloud Computing, Wireless Sensor Network (WSN), Network Security, Neural Network, Artificial Intelligence and MANET (Mobile Ad-Hoc network)..

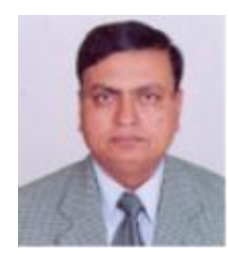

Dr. S.B. Batra is engaged as Dean (academic) with IIST, Rajpur, Mehsana, Gujarat, India. Heholds long experience of working with Indian AirForce Aeronautical Engineer (Electronics).After obtaining Pre-mature retirement in 1999, he is engaged in the field of academic. He has served number of professional universities/engineering collages in India and abroad as director/principal/dean/professor. His many papers are published in various national/international journals. 